\title{
An SU(6) Mott insulator of an atomic Fermi gas realized by large- spin Pomeranchuk cooling
}

\section{$\operatorname{AUTHOR}(\mathrm{S}):$}

Taie, Shintaro; Yamazaki, Rekishu; Sugawa, Seiji; Takahashi, Yoshiro

\section{CITATION:}

Taie, Shintaro ... [et al]. An SU(6) Mott insulator of an atomic Fermi gas realized by largespin Pomeranchuk cooling. Nature Physics 2012, 8(11): 825-830

\section{ISSUE DATE:}

2012-09-23

URL:

http://hdl.handle.net/2433/201987

\section{RIGHT:}

@ 2012 Macmillan Publishers Limited.; この論文は出版社版でありませ ん。引用の際には出版社版をご確認ご利用ください。; This is not the published version. Please cite only the published version. 


\title{
An $\mathrm{SU}(\mathcal{N})$ Mott insulator of an atomic Fermi gas realized by large-spin Pomeranchuk cooling
}

\author{
Shintaro Taie, ${ }^{1, *}$ Rekishu Yamazaki, ${ }^{1,2}$ Seiji Sugawa, ${ }^{1}$ and Yoshiro Takahashi, ${ }^{1,2}$ \\ ${ }^{1}$ Department of Physics, Graduate School of Science, Kyoto University, Japan 606-8502 \\ ${ }^{2}$ CREST, JST, 4-1-8 Honcho Kawaguchi, Saitama 332-0012, Japan
}

(Dated: November 26, 2015)

\begin{abstract}
The Hubbard model, containing only the minimum ingredients of nearest neighbor hopping and on-site interaction for correlated electrons, has succeeded in accounting for diverse phenomena observed in solid-state materials. One of the interesting extensions is to enlarge its spin symmetry to $\mathrm{SU}(\mathcal{N}>2)$, which is closely related to systems with orbital degeneracy. Here we report a successful formation of the $\mathrm{SU}(6)$ symmetric Mott insulator state with an atomic Fermi gas of ytterbium $\left({ }^{173} \mathrm{Yb}\right)$ in a three-dimensional optical lattice. Besides the suppression of compressibility and the existence of charge excitation gap which characterize a Mott insulating phase, we reveal an important difference between the cases of $\mathrm{SU}(6)$ and $\mathrm{SU}(2)$ in the achievable temperature as the consequence of different entropy carried by an isolated spin. This is analogous to Pomeranchuk cooling in solid ${ }^{3} \mathrm{He}$ and will be helpful for investigating exotic quantum phases of $\mathrm{SU}(\mathcal{N})$ Hubbard system at extremely low temperatures.
\end{abstract}

\footnotetext{
* Electronic address: taie@scphys.kyoto-u.ac.jp
} 
In the last decade, a great deal of progress has been made for two-component atomic Fermi gases. High controllability and simplicity for these systems allow systematic study over extremely wide range of system parameters, including interatomic interactions. One of the milestone experiments in strongly correlated regime are the recently reported realization of a fermionic Mott insulator [1,2] for atoms in optical lattices, which is of interest itself and also the parent state of high- $T_{c}$ superconductors [3].

On the other hand, many-body physics with multi-component Fermi gases is experimentally unexplored despite of increasing theoretical interests [4-11]. Fermionic isotopes of alkaline-earth-metal like atoms, such as ytterbium $\left({ }^{173} \mathrm{Yb}\right)[12]$ and strontium $\left({ }^{87} \mathrm{Sr}\right)[13,14]$, are suitable for this aim because of their simple $\mathrm{SU}(\mathcal{N}=2 I+1)$ symmetric interactions for nuclear spin $I[5,9,10] . \mathcal{N}$-component Fermi gas with $\mathrm{SU}(\mathcal{N})$ symmetry in an optical lattice is well described by the $\mathrm{SU}(\mathcal{N})$ Hubbard model

$$
H=-t \sum_{\langle i, j\rangle, \sigma}\left(c_{i, \sigma}^{\dagger} c_{j, \sigma}+\text { H.c. }\right)+\frac{U}{2} \sum_{i, \sigma \neq \sigma^{\prime}} n_{i, \sigma} n_{i, \sigma^{\prime}}+\sum_{k=x, y, z} V_{k} \sum_{i, \sigma}\left(\frac{k_{i}}{d}\right)^{2} n_{i, \sigma},
$$

where $c_{i, \sigma}$ is fermionic annihilation operator for site $i$ and spin $\sigma=-I, \cdots,+I, n_{i, \sigma}=$ $c_{i, \sigma}^{\dagger} c_{i, \sigma}$ is the number operator, $V_{k}=m \omega_{k}^{2} d^{2} / 2$ is the strength of harmonic confinement along $k(=x, y, z)$-axis with an atomic mass $m$ and trap frequency $\omega_{k}$, and $d$ denotes the lattice constant. All parameters, hopping matrix element $t$, on-site interaction $U$ and confinement $V_{k}$ are independent of spin states, which manifests the $\mathrm{SU}(\mathcal{N})$ symmetry of the system. Positive scattering lengths $a=10.55 \mathrm{~nm}$ for ${ }^{173} \mathrm{Yb}$ [15] and $a=5.09 \mathrm{~nm}$ for ${ }^{87} \mathrm{Sr}$ [16] correspond to repulsive interactions $(U>0)$, the case of interest in the context of most theoretical studies. Low temperature behavior of the $\operatorname{SU}(\mathcal{N}>2)$ model is predicted to be qualitatively different from that of SU(2) model, mainly due to the enhancement of quantum fluctuation for large $\mathcal{N}$ system [17]. For instance, the analysis on the square lattice, often considered in the Heisenberg limit $U / t \rightarrow \infty$, reveals the tendency toward disordered spin states $[8,17-21]$, whereas the ground state of the half-filled $\mathrm{SU}(2)$ model is widely believed to be Néel-ordered. Moreover, a striking difference is theoretically predicted for a onedimensional system. An infinitely small repulsive interaction results in a formation of a Mott insulating state for $\mathrm{SU}(2)$ system, whereas a finite strength is required for $\mathrm{SU}(\mathcal{N}>2)$ $[22,23]$. Experimental study of $\mathrm{SU}(\mathcal{N}>2)$ system will lead to better understanding of the underlying physics, and also will provide insights into the important role of the orbital degeneracy $[24,25]$ in condensed matter physics. 
A milestone in the study of the $\mathrm{SU}(\mathcal{N}>2)$ Hubbard system is the realization and characterization of the $\mathrm{SU}(\mathcal{N})$ Mott insulating phase. There are several signatures for a successful formation of a Mott insulating state. One important feature is the existence of the charge excitation gap. As the interaction $U$ increases, the density of states at the Fermi level is decreased, which suppresses the mobility of atoms. Finally the system enters the incompressible Mott phase when the gap opens. At the same time, multiple occupation of lattice sites becomes energetically unfavorable and suppressed in the Mott regime. More quantitatively, fraction of atoms in doubly occupied lattice sites (double occupancy) is closely related to the compressibility $\partial n / \partial \mu$ evaluated at the trap center [26]. Besides these common features of a Mott insulator, it is especially important to clarify the difference between the behaviors of Mott insulators with $\mathrm{SU}(2)$ and $\mathrm{SU}(\mathcal{N}>2)$ symmetries.

Here, we report a successful formation of the $\mathrm{SU}(\mathcal{N}=6)$ symmetric Mott insulator state with an six-spin component atomic Fermi gas of ${ }^{173} \mathrm{Yb}$ in a three-dimensional optical lattice. From double occupancy measurements with photoassociation spectroscopy and lattice modulation spectroscopy, we confirm the above characteristics of the Mott state. Precise control of the spin degrees of freedom provided by optical pumping enables us a straightforward comparison between the cases of $\mathrm{SU}(6)$ and $\mathrm{SU}(2)$. We find an important difference that a lower temperature is obtained for SU(6) Mott insulator as the consequence of larger entropy carried by isolated spin [9, 27] (See also discussions in Section II). In particular, at the lowest temperature achieved, the entropy density at the center of the trap reaches $\ln (6)$, which originates from spin degrees of freedom. Our experimental results are in good agreement with a theoretical calculation based on a high-temperature series expansion (HTSE) which is reliable in the parameter regime of the current experiment [26-28] and a local density approximation (LDA) accompanied with continuum approximation to take into account the presence of the harmonic confinement (See Methods). This work is an important first step and opens the door to the new frontier of the study of strongly correlated phases of the $\mathrm{SU}(\mathcal{N}>2)$ system.

Experimental procedure is as follows (see also Methods for details). The sample is prepared by loading evaporatively cooled Fermi gas of ${ }^{173} \mathrm{Yb}$ into the optical lattices with simple cubic geometry. The initial temperature before loading to the lattice is around $20 \%$ of the Fermi temperature $T_{\mathrm{F}}$. In the following sections, we specify the initial condition in terms of corresponding entropy per particle $s$, in the unit of the Boltzmann constant $k_{\mathrm{B}}$. Double oc- 
cupancy is measured using photoassociation (PA) technique $[29,30]$. We focus on the Mott phase with unit filling, namely one atom per lattice site, and average density at the trap center is below 2 for all experiments presented here. In this case, we can neglect multiple occupation $n_{i} \geq 3$ and double occupancy is simply related to the atom loss $N_{\text {loss }}$ induced by PA, as $D=N_{\text {loss }} / N$ where $N$ is the total atom number without PA.

\section{LATTICE MODULATION SPECTROSCOPY OF SU(6) FERMIONS}

First, we present the experimental evidence of the gap of the SU(6) Mott insulator. The gap can be directly probed by periodically modulating the lattice depth, which induces resonant tunneling to the occupied lattice sites at the modulation frequency close to the Mott gap $\sim U[1]$. This kind of tunneling is detected as the increase in double occupancy. Figure 1 shows the change in double occupancy after lattice modulation, measured at several lattice depths. Here we apply lattice modulation $V(\tau)=V_{0}+\delta V \sin \left(2 \pi f_{\mathrm{m}} \tau\right)$ with a duration $0.4 h / t$. Modulation amplitudes $\delta V$ are chosen to set a perturbation strength $F=\delta t / t-\delta U / U[31]$ to be -0.30 . The Hubbard parameters $t$ and $U$ are calculated using the formulae given by Gerbier et al [32]. The Mott gaps are clearly observed especially at higher lattice depths. For the lattices of $V_{0} \geq 9 E_{\mathrm{R}}$, we find that the observed spectrum is well fitted by Gaussian and the peak positions agree with the calculated value of $U / h$ within $3 \%$.

Lattice modulation spectroscopy does not only give information about excitation spectrum but also correlation between nearest-neighbor lattice sites [33]. From the perturbative treatment for the time evolution of the system under lattice modulation [33-36], we obtain the sum rule about the doublon production rate (DPR) $\Gamma\left(f_{\mathrm{m}}\right)=h / t \partial D / \partial \tau$

$$
\int \Gamma\left(f_{\mathrm{m}}\right) \mathrm{d} f_{\mathrm{m}}=12 \pi^{2} F^{2} \frac{t}{h} \mathcal{P}
$$

where $\mathcal{P}=N^{-1} \sum_{\langle i, j\rangle} \mathcal{P}_{i j}$ is the system averaged correlator. In the case of low filling $n \lesssim 1$ with strong repulsive interaction, the nearest neighbor correlator $\mathcal{P}_{i j}$ is expressed as

$$
\mathcal{P}_{i j}=\sum_{\sigma \neq \bar{\sigma}}\left\langle n_{i, \sigma} \prod_{\sigma^{\prime} \neq \sigma}\left(1-n_{i, \sigma^{\prime}}\right) n_{j, \bar{\sigma}} \prod_{\bar{\sigma}^{\prime} \neq \bar{\sigma}}\left(1-n_{j, \bar{\sigma}^{\prime}}\right)\right\rangle,
$$

where spins $\left(\sigma, \sigma^{\prime}, \bar{\sigma}, \bar{\sigma}^{\prime}\right)$ take $\mathcal{N}$ values. For small modulation amplitudes, observed DPR shows quadratic dependence on $F$, which justifies the use of above linear response theory. 


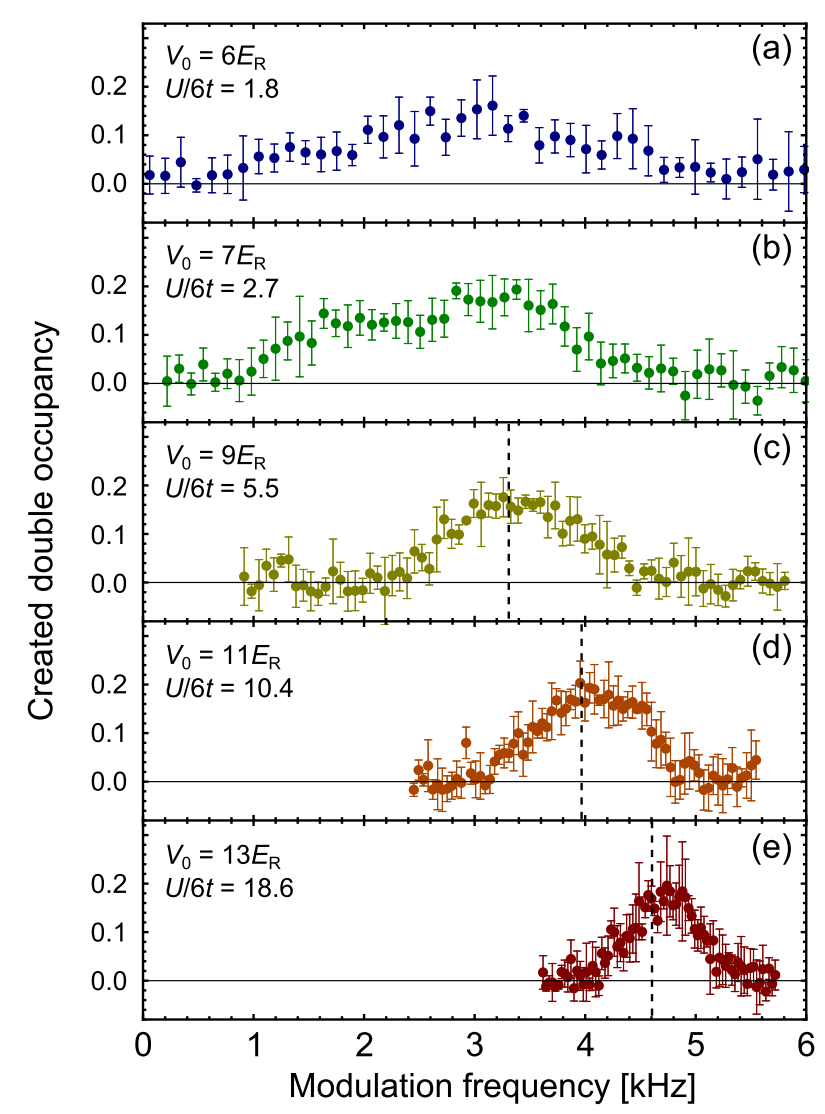

FIG. 1. Lattice modulation spectroscopy. Lattice Modulation spectra obtained for samples with $N=1.9(1) \times 10^{4}$ and $s_{\text {init }}=1.9(2)$, modulation time of $0.4 h / t$, and amplitudes of $\delta V / V_{0}=$ $0.125,0.115,0.10,0.090,0.085$ for lattice depths of $6,7,9,11,13$ in unit of $E_{\mathrm{R}}$, respectively. The vertical dashed lines indicate the calculated values of on-site interaction $U / h$ for the corresponding lattice depth. The error bars denote s.d. of the measurements.

In the high-temperature regime $T \gg t^{2} / U$, we can neglect the spin correlation between adjacent sites and the above expression for $\mathcal{P}_{i j}$ reduces to

$$
\mathcal{P}_{i j}=\frac{\mathcal{N}-1}{\mathcal{N}} W_{i}(1) W_{j}(1)
$$

where $W_{i}(1)$ denotes the probability of single occupation at site $i$. The factor $(\mathcal{N}-1) / \mathcal{N}$ is nothing more than the effect of Pauli exclusion on the hopping process. Compared with the case of $\mathrm{SU}(2)$, larger DPR is expected for an $\mathrm{SU}(6)$ Mott insulator because of factor $5 / 3$ enhancement due to the reduction of the Pauli exclusion effect. On the other hand, the thermodynamic properties are reflected in the correlator through $W_{i}(1)$, which increases with lowering temperature and approaches unity when the system becomes a defect-free 
Mott insulator with unit filling.

(a)

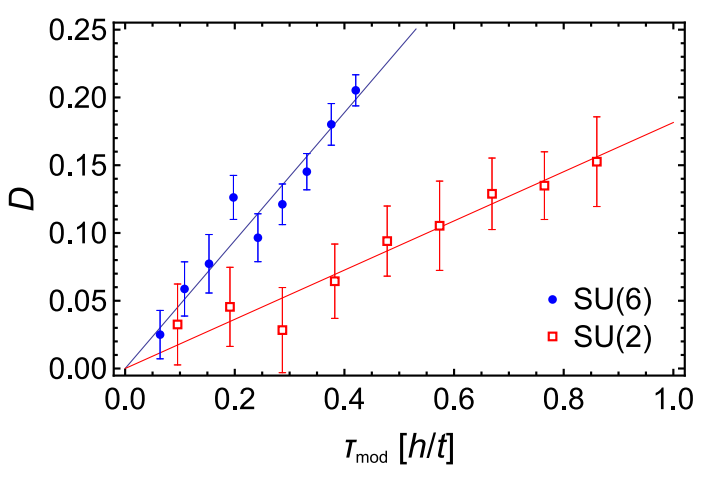

(b)

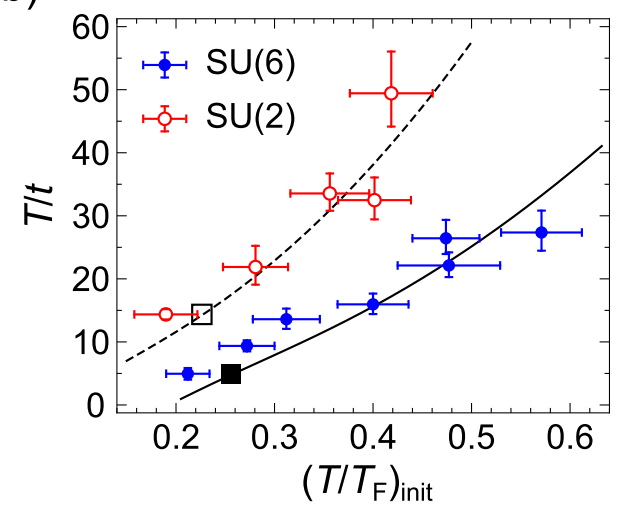

(c)

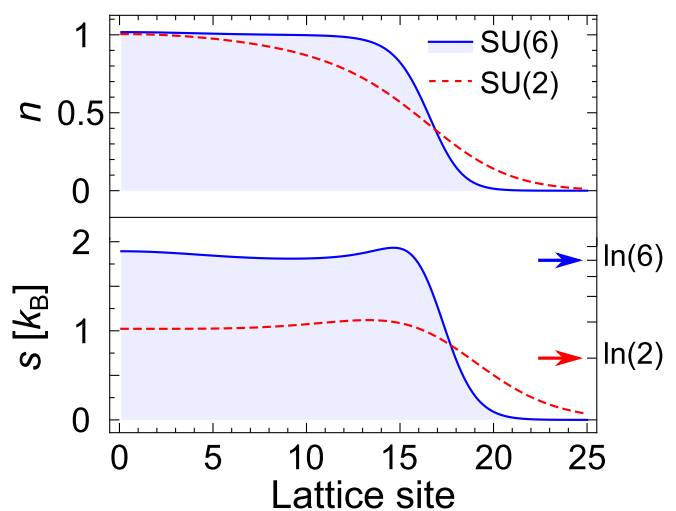

FIG. 2. Doublon production rate for $\mathbf{S U}(\mathcal{N})$ Mott insulators. (a) Measurement of the doublon production rate. Linear increase is observed for short modulation times presented here. Atom number is $N=1.9(1) \times 10^{4}$, the lattice depth is $11 E_{\mathrm{R}}(t / h=63.7 \mathrm{~Hz}$ and $U / h=4.0$ $\mathrm{kHz}$ ), and the modulation amplitude $\delta V / V_{0}$ is $9.0 \%$. Linear fits yield the values $\Gamma=0.47(2)$ and 0.18(1) for the case of SU(6) and SU(2), respectively. Error bars denote s.e. for 10-15 independent measurements. (b) Temperatures of SU(6) (blue circles) and SU(2) (open red circles) Fermi gases in the lattice inferred from the measured DPR. The dependence on the initial temperature in the harmonic trap is shown. The solid and dashed lines are the corresponding theoretical curves which assume adiabatic loading into the lattice. Error bars include the fitting error in determining DPR and s.d. of initial temperatures. (c) Calculated density (top) and entropy distribution (bottom) at the lowest measured temperatures for 6- and 2-component cases, indicated by square symbols in the graph (a). Maximum spin entropy $\ln (\mathcal{N}=6,2)$ are indicated by the arrows. 


\section{COMPARISON BETWEEN SU(6) AND SU(2) FERMIONS}

We measure the DPR at the peak of the modulation spectrum, both for SU(6) and $\mathrm{SU}(2)$ Mott insulators, as shown in Fig. 2 (a) (see also supplementary information S1). Here we produce $\mathrm{SU}(2)$ symmetric system of ${ }^{173} \mathrm{Yb}$ by optical pumping [37] (See Methods), in order to make a direct experimental comparison between the behaviors of $\mathrm{SU}(6)$ and $\mathrm{SU}(2)$ systems. The modulation spectra are well fitted with a Gaussian shape of $e^{-2}$ full width of $25(2) t / h$, determined from the spectrum for modulation time $\tau=0.3 h / t$ (see Fig. S1 (a)). The peak DPR is extracted from linear fitting to the data taken for several modulation times. The measured DPR for an SU(6) Mott insulator exceeds 5/3 of that for SU(2), which indicates the lower temperature is realized (Fig. 2 (a)). This is clarified in Fig. 2 (b), where we plot the temperature in the lattice determined from the measured DPR. Temperature estimation is based on the formalism presented in the previous section and the calculation of the quantity $W_{i}(1)$ from the high-temperature series expansion (HTSE), as described in Methods. The temperatures for SU(6) Fermi gases are the factor of about 2-3 smaller than that for the $\mathrm{SU}(2)$ cases. We also show in Fig. 2 (c) the density and entropy distributions calculated for the case of the largest DPR, in other words, the lowest temperature achieved. In the case of $\mathrm{SU}(6)$, a robust Mott plateau is clearly formed and entropy per site is very close to $\ln (6)$. We note that, in a trapped system, entropy is pushed onto a metallic state near the edge of the cloud and a Mott insulator at the trap center survives for higher total entropy [2]. On the contrary, starting from the almost same initial $T / T_{\mathrm{F}}$ in a harmonic trap before loading into the lattice, the plateau has been largely melted for the $\mathrm{SU}(2)$ case.

The observed striking difference between the temperatures required for accessing the Mott phase of $\mathrm{SU}(2)$ and $\mathrm{SU}(\mathcal{N}>2)$ cases can be understood by a following simple argument, as shown in Fig. 3. In the atomic limit where tunneling between lattice sites is neglected, the maximum spin entropy per atom is equal to $\ln \mathcal{N}$ for an $\mathcal{N}$-component Fermi gas in the Mott state with unit filling. On the other hand, motional degrees of freedom must be frozen in the Mott phase. Assuming adiabaticity of loading process into optical lattices, we can expect that the defect-free Mott phase in an optical lattice forms at $\left(T / T_{\mathrm{F}}\right)_{\text {init }} \sim \ln \mathcal{N} / \pi^{2}$ where $\left(T / T_{\mathrm{F}}\right)_{\text {init }}$ is measured in a harmonic trap before loading into lattices. This implies a significant reduction of the required initial temperature in the unit of $T_{\mathrm{F}}$ for large $\mathcal{N}$ system, as predicted in Refs. $[9,27]$. In other words, large spin can effectively cool down the system 


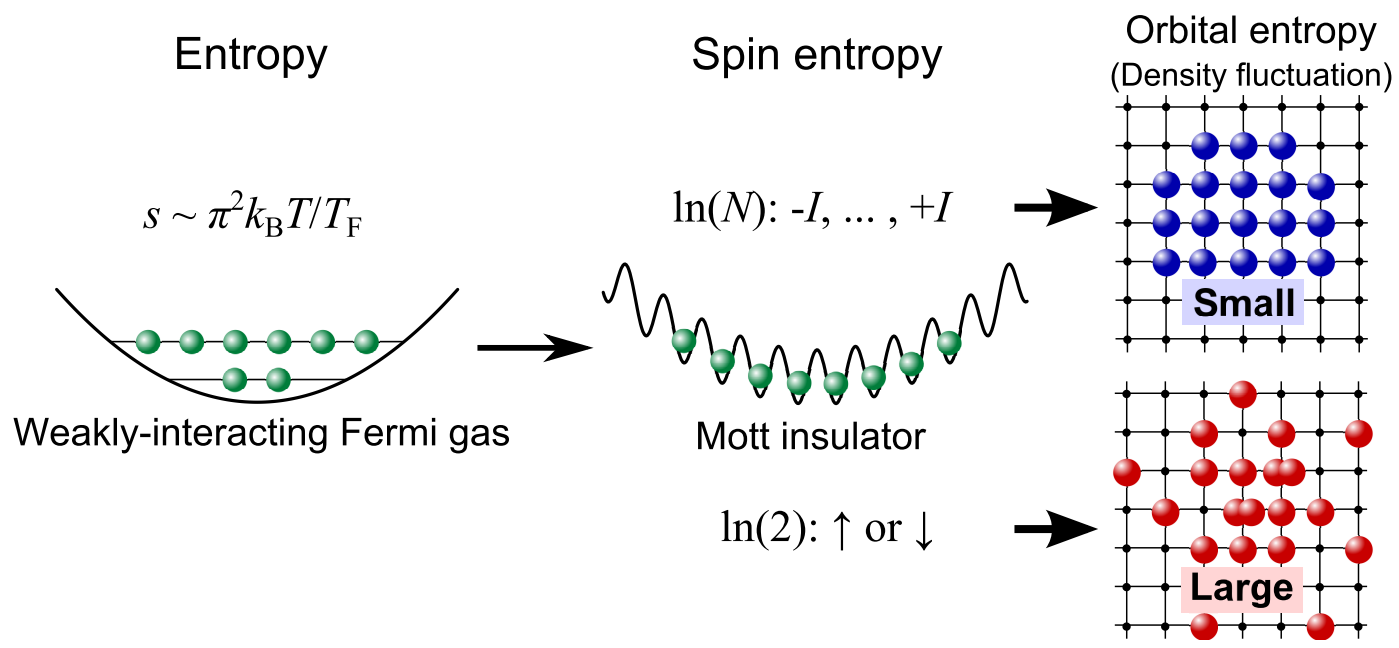

FIG. 3. Schematic of enhanced Pomeranchuk cooling in an $\mathrm{SU}(\mathcal{N})$ Fermi gas. When the system evolves from a weakly-interacting degenerate Fermi gas into a Mott insulator, spin degrees of freedom arise. Lager entropy can be absorbed by isolated spins of an $\mathrm{SU}(\mathcal{N}>2)$ Mott insulator, resulting in the reduction of density fluctuation which has dominant contribution in determining the temperature. In real experiments, the behavior of absolute temperature strongly depends on the harmonic confinement: the system can even be heated if strong compression occurs during loading into the lattice. However, the general trend that larger $\mathcal{N}$ leads to lower temperature remains unchanged.

by absorbing entropy from motional degrees of freedom, which is the same mechanism as Pomeranchuk cooling observed in solid ${ }^{3} \mathrm{He}[38]$.

Although this mechanism was responsible for achieving novel quantum phases in a mixed gas of bosons and fermions in a recent report [30], no systematic study was done on the detailed behaviors of enhanced Pomeranchuk cooling, especially the comparison between the $\mathrm{SU}(2)$ and $\mathrm{SU}(6)$ which is clearly demonstrated in this work.

\section{DOUBLE OCCUPANCY MEASUREMENT OF SU(6) FERMIONS}

Double occupancy measurement has been extensively used as the probe for fermionic lattice systems $[1,2,39]$ to obtain the information on the compressibility. While the correlation measurement presented above is a good probe for the low density case $n \leq 1$, the double occupancy is suitable for characterizing states with high filling, especially $1 \leq n \leq 2$. Figure 4 
(a)

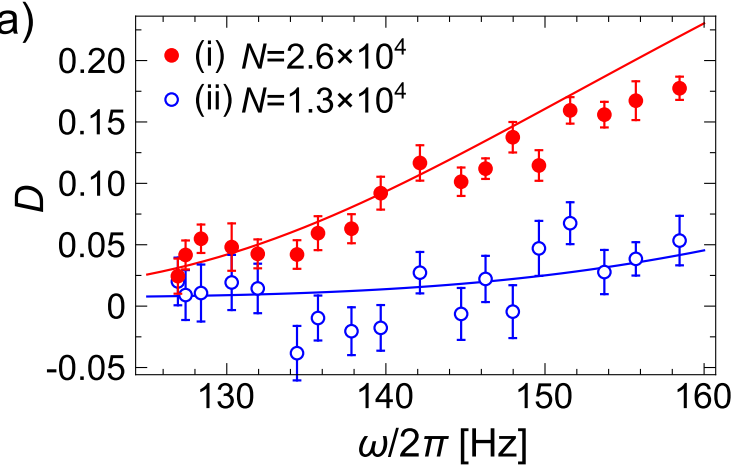

(b)

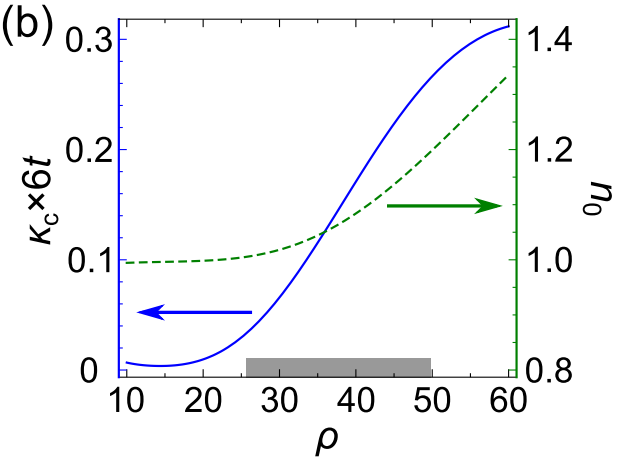

FIG. 4. Double occupancy and compressibility. (a) Measured double occupancy. Two data series with different atom number (i) $N=2.6(1) \times 10^{4}$ (red circles) and (ii) $1.3(1) \times 10^{4}$ (open blue circles) are shown. The solid lines are the best fitted theoretical curves according to the second order high-temperature expansion, corresponding $s=2.2$ and 2.5 for (i) and (ii), respectively. The error bars denote s.e. for typically 15 independent measurements. (b) Calculated central compressibility $\kappa_{\mathrm{c}}$ (solid) as the function of characteristic filling $\rho$, together with the central density $n_{\mathrm{c}}$ (dashed) with the parameters for data (ii) in (a). Fixed entropy $s=2.5$ obtained by the fit in (a) is used. Experimentally relevant range of $\rho$ is indicated by the shaded region.

(a) shows the measured double occupancy for various mean trap frequencies $\omega=\left(\omega_{x} \omega_{y} \omega_{z}\right)^{1 / 3}$, at the lattice depth of $9.0 E_{\mathrm{R}}$. Corresponding Hubbard parameters are given as $t / h=101 \mathrm{~Hz}$ and $U / h=3.3 \mathrm{kHz}$. The data are taken for two initial preparations of (i) $N=2.6(1) \times 10^{4}$ and $s_{\text {init }}=1.7(1)$ and (ii) $N=1.3(1) \times 10^{4}$ and $s_{\text {init }}=1.9(3)$. For the data (i), the double occupancy increases with tighter confinement and the trap center is metallic with $n>1$. On the other hand, the data (ii) shows that the double occupancy is essentially zero for low compression regime, indicating the formation of an $n=1$ Mott plateau. We fit the data to a theoretical curve based on the HTSE by taking the entropy per particle in the lattice $s$ as only one fitting parameter. We then obtain $s=2.2$ and 2.5 for the set (i) and (ii), respectively, which are slightly higher than $s_{\text {init }}$. Since they still lie between $s_{\text {init }}$ and the entropies measured after reversed loading into the harmonic trap which range from 2.8 to 3.4, the deduced values of entropy $s$ are reasonable and consistent with the existence of some constant heating or non-adiabatic effects during the loading and reverse loading processes.

Incompressibility due to the repulsive interaction, which characterizes a Mott insulator, can be deduced from the obtained entropy in the lattice. In Fig. 4 (b) we show the calculated 
density $n_{0}$ and compressibility at the trap center $\kappa_{\mathrm{c}}=\partial n_{0} / \partial \mu$ as a function of characteristic filling defined as $\rho=N\left(12 t / m \omega^{2} d^{2}\right)^{-3 / 2}$. Introducing characteristic filling enables to unify the data with different total atom number and the harmonic confinement, within the LDA formalism $[28,40]$. For the lowest measured $\rho \sim 25, n_{0}$ is almost unity and $\kappa_{\mathrm{c}}$ is as low as $0.03 / 6 t$, which means that the system is deep in the strongly incompressible Mott insulating regime. We note that this value of $\kappa_{0}$ is a factor of $\sim 3$ smaller than that expected in the case of the $\mathrm{SU}(2)$ Hubbard model with the same parameters $(s, \rho$, and $U / t)$, as the consequence of large-spin Pomeranchuk cooling mentioned above.

This work clearly shows the realization of an SU(6) Mott insulator with unit filling and demonstrates the existence of enhanced Pomeranchuk cooling for large spin systems. Pomeranchuk cooling is most effective for systems with low filling for which atoms possesses full spin degrees of freedom. We note that it also works for higher fillings because (a) entropy per atom $n^{-1} \ln \left({ }_{\mathcal{N}} \mathrm{C}_{n}\right)$ is larger than $\ln (2)$ for $n \leq \mathcal{N} / 2$ and (b) a Mott plateau with high filling is surrounded by shells with lower filling, where spin degree of freedom survives.

\section{Note added.}

After completion of our work, we noticed some theoretical works [41-43] which study thermodynamics of $\mathrm{SU}(\mathcal{N})$ Hubbard/Heisenberg model. They found that the advantage of large- $\mathcal{N}$ systems in adiabatic cooling, which is demonstrated in our experiment, is still valid in low temperature regime $\left(k_{\mathrm{B}} T \sim t^{2} / U\right)$ where magnetic correlations take place.

\section{METHODS}

\section{A. Preparation of a degenerate fermi gas of ${ }^{173} \mathrm{Yb}$ with 6 spin components}

The method for preparing a degenerate Fermi gas of ${ }^{173} \mathrm{Yb}$ is essentially the same as that described before [12] except that the detailed parameters of the experimental apparatus have been changed (For current setup, see also Ref. [44]). A balanced mixture of all nuclear spin states is evaporatively cooled in a crossed far-off resonant optical trap (FORT). At the end of evaporation, we have $1-3 \times 10^{4}$ atoms with a temperature around $0.2 T_{\mathrm{F}}$. Temperature is obtained by performing standard Thomas-Fermi fitting to the observed momentum distributions and entropy per particle is calculated from measured $T / T_{\mathrm{F}}$ using the formula for 
non-interacting Fermi gas. The spin distribution is measured by an optical Stern-Gerlach effect and confirmed to be equal within 5\% relative uncertainty [37]. Atoms are subsequently loaded into an optical lattice within $150 \mathrm{~ms}$. The lattice potential is formed by three mutually orthogonal laser beams with the lattice constant $d=266 \mathrm{~nm}$. During the first 100 ms of loading process, the lattice is ramped up to $5 E_{\mathrm{R}}$ and at the same time we change the power of the FORT laser to obtain desired confinement strength. The lattice depth is calibrated by analyzing diffraction patterns of a Bose-Einstein condensate of ${ }^{174} \mathrm{Yb}$ after the application of the pulsed lattice potential [45]. Trap frequencies are determined from dipole oscillations. For lattice modulation experiments (Fig. 1 and 2), the mean trap frequencies $\omega / 2 \pi$ are $115.2(8), 119.6(9), 127.4(10), 134.5(11), 140.9(13)[\mathrm{Hz}]$ for the lattice depths of $6,7,9,11,13$ in unit of $E_{\mathrm{R}}$, respectively.

\section{B. Preparation of a degenerate Fermi gas of ${ }^{173} \mathrm{Yb}$ with 2 spin components}

At the early stage of evaporative cooling, atoms are pumped into $m_{F}=+5 / 2$ and $-5 / 2$ states by $\pi$-polarized laser light which is resonant with the ${ }^{1} S_{0} \leftrightarrow{ }^{3} P_{1}\left(F^{\prime}=3 / 2\right)$ transition. The fraction of residual spin components are estimated to be $N_{m} \leq 0.03 N_{+5 / 2}$ for $m=-3 / 2, \cdots+3 / 2$. We observe no detectable spin changing collision such as $m_{F}=$ $(+5 / 2,-5 / 2) \rightarrow(+3 / 2,-3 / 2)$ during several second of evaporation, which is the characteristic of $\mathrm{SU}(\mathcal{N})$ symmetry $[10]$.

\section{Measurement of doubly occupied site by photoassociation}

First, the lattices are ramped up to desired depth for which Mott insulating states can be reached. We then increase the lattice depth to $25 E_{\mathrm{R}}$ in $2 \mathrm{~ms}$ to suppress tunneling during the measurement, followed by $10 \mathrm{~ms}$ irradiation of PA laser light. The PA laser is detuned by $-796 \mathrm{MHz}$ from the ${ }^{1} S_{0} \leftrightarrow{ }^{3} P_{1}$ atomic transition $(\lambda=556 \mathrm{~nm})$ and has the intensity of $\sim 0.5 \mathrm{~W} / \mathrm{cm}^{2}$. The PA process enables to convert all atoms on doubly occupied sites into electronically excited molecules which rapidly escape from the trap. The loss of atom is, therefore, the measure of the double occupancy. 


\section{Theoretical method}

Theoretical calculations presented in this paper are based on high-temperature expansion of the SU(6) Hubbard model [27, 46, 47]. Extending the calculation in Ref. [47] to the $\mathrm{SU}(\mathcal{N})$ case, we have the grand-canonical free energy per lattice site up to second order in $t / k_{\mathrm{B}} T=\beta t:$

$$
\begin{aligned}
\Omega(T, \mu)= & \Omega_{0}(T, \mu)+\Delta \Omega \\
-\beta \Delta \Omega= & \left(\frac{\beta t}{Z_{0}}\right)^{2} z \mathcal{N}\left[\frac{1}{2} \sum_{n_{1}=1}^{\mathcal{N}}\left(\begin{array}{l}
\mathcal{N}-1 \\
n_{1}-1
\end{array}\right)^{2} x^{2 n_{1}-1} w^{\left(n_{1}-1\right)^{2}}\right. \\
& \left.-\frac{1}{\beta U} \sum_{n_{1} \neq n_{2}}^{\mathcal{N}}\left(\begin{array}{l}
\mathcal{N}-1 \\
n_{1}-1
\end{array}\right)\left(\begin{array}{l}
\mathcal{N}-1 \\
n_{2}-1
\end{array}\right) \frac{x^{n_{1}+n_{2}-1} w^{\frac{1}{2} n_{1}\left(n_{1}-1\right)+\frac{1}{2}\left(n_{2}-1\right)\left(n_{2}-2\right)}}{n_{1}-n_{2}}\right],
\end{aligned}
$$

where $z$ is the number of nearest neighbors, $x=\mathrm{e}^{\beta \mu}, w=\mathrm{e}^{-\beta U}$, and $\Omega_{0}$ is the free energy in the atomic limit given by

$$
\begin{aligned}
& \Omega_{0}(T, \mu)=-k_{\mathrm{B}} T \ln Z_{0}(T, \mu), \\
& Z_{0}(T, \mu)=\sum_{n=0}^{\mathcal{N}}\left(\begin{array}{l}
\mathcal{N} \\
n
\end{array}\right) \exp \left[-\beta\left(\frac{U}{2} n(n-1)-\mu n\right)\right] .
\end{aligned}
$$

For a trapped system, we apply local density approximation in which the system is regarded as locally uniform. Thus we have $\Omega_{\mathrm{LDA}}=\sum_{i} \Omega_{i}=\sum_{i} \Omega\left(T, \mu_{i}\right)$, where $\mu_{i}=\mu-V_{i}$ is the local chemical potential for the external potential $V_{i}$. Finally we approximate the sum over lattice sites with spatial integration (continuum approximation).

As our experiments are carried out near unit filling $n=1$ with strong repulsive interaction, we can neglect multiple occupation with filling $n \geq 3$. In this case, double occupancy is given by the same formula as for the $\mathrm{SU}(2)$ model:

$$
D=\frac{2}{N} \sum_{i}\left\langle n_{i, \uparrow} n_{i, \downarrow}\right\rangle=\frac{2}{N} \frac{\partial \Omega_{\mathrm{LDA}}}{\partial U} .
$$

To obtain the expression for the correlator $\mathcal{P}_{i j}$, we use the relation $\left\langle n_{i}\right\rangle=\sum_{n}^{6} n W_{i}(n) \simeq$ $W_{i}(1)+2 W_{i}(2)=-\partial \Omega_{i} / \partial \mu$, where $W_{i}(2)$ is local double occupancy and is given by $\partial \Omega_{i} / \partial U$. Then the correlator is given in terms of the derivatives of the free energy as

$$
\mathcal{P}_{i j}=\frac{\mathcal{N}-1}{\mathcal{N}}\left(\frac{\partial \Omega_{i}}{\partial \mu}+2 \frac{\partial \Omega_{i}}{\partial U}\right)\left(\frac{\partial \Omega_{j}}{\partial \mu}+2 \frac{\partial \Omega_{j}}{\partial U}\right) .
$$




\section{Acknowledgements}

We acknowledge M. A. Cazalilla, T. Giamarchi, V. Gurarie, K. R. A. Hazzard, M. Hermele, K. Inaba, A. M. Rey, A. Tokuno and M. Yamashita for helpful discussions. This work is supported by the Grant-in-Aid for Scientific Research of JSPS (No. 18204035, 21102005C01 (Quantum Cybernetics)), GCOE Program "The Next Generation of Physics, Spun from Universality and Emergence" from MEXT of Japan, and World- Leading Innovative R\&D on Science and Technology (FIRST). ST acknowledge supports from JSPS.

[1] Jördens, R., Strohmaier, N., Günter, K., Moritz, H. \& Esslinger, T. A Mott insulator of fermionic atoms in an optical lattice. Nature 455, 204 (2008).

[2] Schneider, U. et al. Metallic and insulating phases of repulsively interacting fermions in a 3D optical lattice. Science 322, 1520 (2008).

[3] Lee, P. A., Nagaosa, N. \& Wen, X.-G. Doping a Mott insulator: Physics of high-temperature superconductivity. Rev. Mod. Phys. 78, 17 (2006).

[4] Ho, T.-L. \& Yip, S. Pairing of fermions with arbitrary spin. Phys. Rev. Lett. 82, 247 (1999).

[5] Wu, C., Hu, J.-p. \& Zhang, S.-c. Exact $\mathrm{SO}(5)$ symmetry in the spin-3/2 fermionic system. Phys. Rev. Lett. 91, 186402 (2003).

[6] Honerkamp, C. \& Hofstetter, W. Ultracold fermions and the $\mathrm{SU}(N)$ Hubbard model. Phys. Rev. Lett. 92, 170403 (2004).

[7] Cherng, R. W., Refael, G. \& Demler, E. Superfluidity and magnetism in multicomponent ultracold fermions. Phys. Rev. Lett. 99, 130406 (2007).

[8] Hermele, M., Gurarie, V. \& Rey, A. M. Mott insularors of ultracold fermionic alkaline earth atoms: Underconstrained magnetism and chiral spin liquid. Phys. Rev. Lett. 103, 135301 (2009).

[9] Cazalilla, M. A., Ho, A. F. \& Ueda, M. Ultracold gases of ytterbium ferromagnetism and Mott states in an SU(6) Fermi system. New J. Phys. 11, 103033 (2009).

[10] Gorshkov, A. V. et al. Two-orbital SU(N) magnetism with ultracold alkaline-earth atoms. Nature Phys. 6, 289 (2010). 
[11] Yip, S.-K. Bose-Einstein condensation in the presence of artificial spin-orbit interaction. Phys. Rev. A 83, 043616 (2011).

[12] Fukuhara, T., Takasu, Y., Kumakura, M. \& Takahashi, Y. Degenerate Fermi gases of ytterbium. Phys. Rev. Lett. 98, 030401 (2007).

[13] DeSalvo, B. J., Yan, M., Mickelson, P. G., Martinez de Escobar, Y. N. \& Killian, T. C. Degenerate Fermi gas of ${ }^{87}$ Sr. Phys. Rev. Lett. 105, 030402 (2010).

[14] Tey, M. K., Stellmer, S., Grimm, R. \& Schreck, F. Double-degenerate Bose-Fermi mixture of strontium. Phys. Rev. A 82, 011608 (2010).

[15] Kitagawa, M. et al. Two-color photoassociation spectroscopy of ytterbium atoms and the precise determinations of $s$-wave scattering lengths. Phys. Rev. A 77, 012719 (2008).

[16] Martinez de Escobar, Y. N. et al. Two-photon photoassociative spectroscopy of ultracold ${ }^{88} \mathrm{Sr}$. Phys. Rev. A 78, 062708 (2008).

[17] Wu, C. Hidden symmetry and quantum phases in spin-3/2 cold atomic systems. Mod. Phys. Lett. B 20, 1707 (2006).

[18] Affleck, I. \& Marston, J. B. Large- $n$ limit of the Heisenberg-Hubbard model: Implications for high- $T_{c}$ superconductors. Phys. Rev. B 37, 3774-3777 (1988).

[19] Read, N. \& Sachdev, S. Some features of the phase diagram of the square lattice $\mathrm{SU}(N)$ antiferromagnet. Nucl. Phys. B 316, 609 (1989).

[20] Kawashima, N. \& Tanabe, Y. Ground states of the SU(N) Heisenberg model. Phys. Rev. Lett. 98, 057202 (2007).

[21] Hermele, M. \& Gurarie, V. Topological liquids and valence cluster states in two-dimensional SU(N) magnets. Phys. Rev. B 84, 174441 (2011).

[22] Assaraf, R., Azaria, P., Caffarel, M. \& Lecheminant, P. Metal-insulator transition in the one-dimensional SU(N) Hubbard model. Phys. Rev. B 60, 2299-2318 (1999).

[23] Manmana, S. R., Hazzard, K. R. A., Chen, G., Feiguin, A. E. \& Rey, A. M. SU(N) magnetism in chains of ultracold alkaline-earth-metal atoms: Mott transitions and quantum correlations. Phys. Rev. A 84, 043601 (2011).

[24] Li, Y. Q., Ma, M., Shi, D. N. \& Zhang, F. C. SU(4) theory for spin systems with orbital degeneracy. Phys. Rev. Lett. 81, 3527-3530 (1998).

[25] Tokura, Y. \& Nagaosa, N. Orbital physics in transition-metal oxides. Science 288, 462-468 (2000). 
[26] Scarola, V. W., Pollet, L., Oitmaa, J. \& Troyer, M. Discerning incompressible and compressible phases of cold atoms in optical lattices. Phys. Rev. Lett. 102, 135302 (2009).

[27] Hazzard, K. R. A., Gurarie, V., Hermele, M. \& Rey, A. M. High-temperature properties of fermionic alkaline-earth-metal atoms in optical lattices. Phys. Rev. A 85, 041604 (2012).

[28] De Leo, L., Bernier, J.-S., Kollath, C., Georges, A. \& Scarola, V. W. Thermodynamics of the three-dimensional Hubbard model: Implications for cooling cold atomic gases in optical lattices. Phys. Rev. A 83, 023606 (2011).

[29] Rom, T. et al. State selective production of molecules in optical lattices. Phys. Rev. Lett. 93, $073002(2004)$.

[30] Sugawa, S. et al. Interaction and filling-induced quantum phases of dual Mott insulators of bosons and fermions. Nature Phys. 7, 642 (2011).

[31] Reischl, A., Schmidt, K. P. \& Uhrig, G. S. Temperature in one-dimensional bosonic Mott insulators. Phys. Rev. A 72, 063609 (2005).

[32] Gerbier, F. et al. Interference pattern and visibility of a Mott insulator. Phys. Rev. A 72, $053606(2005)$.

[33] Greif, D., Tarruell, L., Uehlinger, T., Jördens, R. \& Esslinger, T. Probing nearest neighbor correlations of ultracold fermions in an optical lattice. Phys. Rev. Lett. 106, 145302 (2011).

[34] Kollath, C., Iucci, A., McCulloch, I. P. \& Giamarchi, T. Modulation spectroscopy with ultracold fermions in an optical lattice. Phys. Rev. A 74, 041604 (2006).

[35] Huber, S. D. \& Rüegg, A. Dynamically generated double occupancy as a probe of cold atom systems. Phys. Rev. Lett. 102, 065301 (2009).

[36] Hassler, F. \& Huber, S. D. Coherent pumping of a Mott insulator: Fermi golden rule versus Rabi oscillations. Phys. Rev. A 79, 021607 (2009).

[37] Taie, S. et al. Realization of a $\mathrm{SU}(2) \times \mathrm{SU}(6)$ system of fermions in a cold atomic gas. Phys. Rev. Lett. 105, 190401 (2010).

[38] Richardson, R. C. The Pomeranchuk effect. Rev. Mod. Phys. 69, 683-690 (1997).

[39] Jördens, R. et al. Quantitative determination of temperature in the approach to magnetic order of ultracold fermions in an optical lattice. Phys. Rev. Lett. 104, 180401 (2010).

[40] Rigol, M. \& Muramatsu, A. Quantum Monte Carlo study of confined fermions in onedimensional optical lattices. Phys. Rev. A 69, 053612 (2004). 
[41] Cai, Z., Hung, H.-h., Wang, L., Zheng, D. \& Wu, C. Pomeranchuk cooling of the SU(2N) ultra-cold fermions in optical lattices. Preprint at arXiv:1202.6323 (2012).

[42] Messio, L. \& Mila, F. Entropy dependence of correlations in one-dimensional SU( $N)$ antiferromagnets. Preprint at arXiv:1207.1320 (2012).

[43] Bonnes, L. et al. Addiabatic loading of one-dimensional $\mathrm{SU}(N)$ alkaline earth fermions in optical lattices. Preprint at arXiv:1207.3900 (2012).

[44] Sugawa, S., Yamazaki, R., Taie, S. \& Takahashi, Y. Bose-Einstein condensate in gases of rare atomic species. Phys. Rev. A 84, 011610 (2011).

[45] Denschlag, J. H. et al. Bose-Einstein condensate in an optical lattice. J. Phys. B: At. Mol. Opt. Phys. 35, 3095 (2002).

[46] ten Haaf, D. F. B. \& van Leeuwen, J. M. J. High-temperature series expansions for the Hubbard model. Phys. Rev. B 46, 6313-6327 (1992).

[47] Henderson, J. A., Oitmaa, J. \& Ashley, M. C. B. High-temperature expansion for the singleband Hubbard model. Phys. Rev. B 46, 6328 (1992). 


\section{Supplementary Information}

\section{S1. MEASUREMENT OF NEAREST NEIGHBOR CORRELATOR}

Within the perturbative formalism used in this paper, we need the frequency-integrated doublon production rate (DPR) to obtain the nearest neighbor correlator. Reference [1] reported that $\Gamma(\hbar \omega)$ has approximately Gaussian shape with $\mathrm{e}^{-2}$ full width of $24 t$ for a trapped $\mathrm{SU}(2)$ Mott insulator. In our case of an $\mathrm{SU}(6)$ Mott insulator, the spectrum has the width of 25(2)t (Figure S1 (a)), which is in good agreement with their result. Given the width of a modulation spectrum, the correlator can be calculated from the DPR measured at the peak of the spectrum. Figure S1 (b) shows the peak DPR measured at several initial temperatures, which is the basis of thermometry presented in Fig. 2 (b) in the main text. Remarkable enhancement of the DPR for SU(6) fermions is observed, which is due to the combined effect of the greater Pauli suppression of tunneling for the SU(2) case and the enhancement of the Pomeranchuk effect for the SU(6) case.

(a)

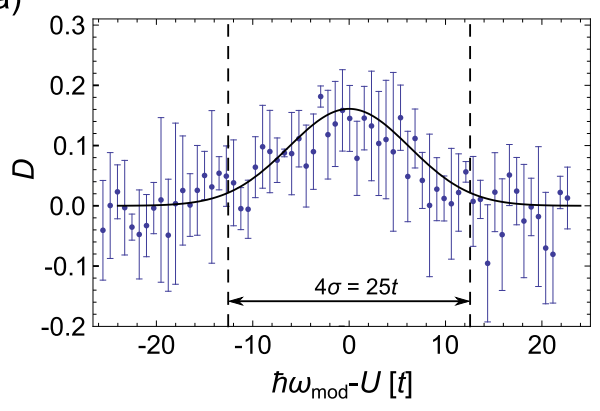

(b)

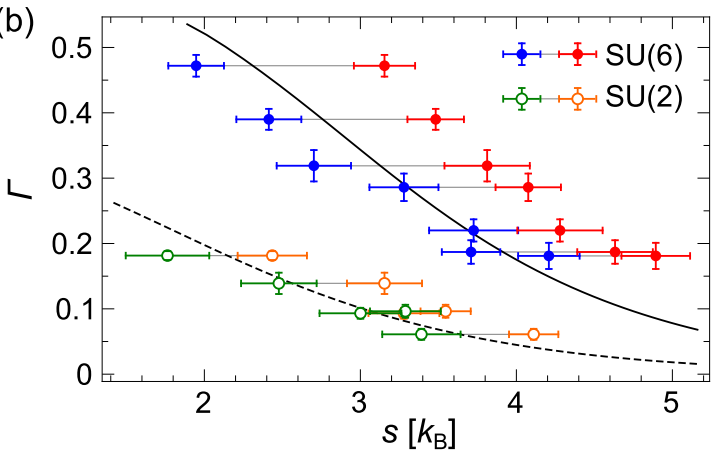

FIG. S 1. (a) Lattice modulation spectrum for the lattice depth of $11 E_{\mathrm{R}}$, the modulation amplitude of $\delta V / V_{0}=9.0 \%$, and modulation time of $0.32 h / t$, which is shorter than that for Fig. 2. We extract the width $4 \sigma=25(2) t$ from a Gaussian fit to the data. Error bars denotes s.d. of the measurements. (b) Peak DPR obtained for both SU(6) (closed circles) and SU(2) (open circles) Mott insulators, as a function of entropy per particle $s$ measured in the harmonic trap. We measure $s$ before loading to the lattice (blue and green) and also after reversing the loading prededure (red and orange), which set the lower and the upper limit, respectively. The corresponding two values are connected by the gray lines. 


\section{S2. EFFECT OF RESIDUAL SPIN COMPONENTS}

Here we discuss the effect of residual population in the $m_{F}= \pm 3 / 2$ and $\pm 1 / 2$ states due to imperfect optical pumping to $m_{F}= \pm 5 / 2$ states in the case of $\mathrm{SU}(2)$ experiments. For a uniform Mott insulator with unit filling, the entropy per atom is given by $s=k_{\mathrm{B}} N^{-1} \ln \left(N ! / \prod_{\sigma} N_{\sigma} !\right)$ where $N$ is the total atom number and $N_{\sigma}$ is the atom number for the spin component $\sigma$. Below we consider the special case that each minority component is equally populated. We define the imbalance parameter $p$ as $N_{-3 / 2}=\cdots=N_{+3 / 2}=$ $p N_{+5 / 2}\left(=p N_{-5 / 2}\right)$. In the case of $p=0.03$ which is upper limit in our experiment, we have $s / k_{\mathrm{B}}=0.96$, considerably larger than $\ln (2)=0.69$ for the pure $\mathrm{SU}(2)$ case. This large spin entropy cools the sample and enhances DPR. In addition, impurity components reduce the effect of Pauli principle in hopping processes, which also contributes to the increase of DPR. We estimate this effect to be $\sim 10 \%$ for $p=0.03$.

For more quantitative argument, we calculate the DPR for six-component gases with imbalanced population within the atomic limit. Imbalanced population leads to two chemical potentials $\mu_{\mathrm{m}}$ and $\mu_{\mathrm{r}}$ for two majority components of $m_{F}= \pm 5 / 2$ and residual minority ones of $m_{F}= \pm 3 / 2, \pm 1 / 2$, respectively. We consider the probability $W_{i}\left(n_{\mathrm{m}}, n_{\mathrm{r}}\right)$ that site $i$ is occupied by $n_{\mathrm{m}}$ majority spins and $n_{\mathrm{r}}$ minority spins. In the atomic limit, it is given by

$$
\begin{aligned}
W_{i}\left(n_{\mathrm{m}}, n_{\mathrm{r}}\right) & =\frac{1}{Z}\left(\begin{array}{c}
2 \\
n_{\mathrm{m}}
\end{array}\right)\left(\begin{array}{c}
4 \\
n_{\mathrm{r}}
\end{array}\right) B_{i}\left(n_{\mathrm{m}}, n_{\mathrm{r}}\right), \\
B_{i}\left(n_{\mathrm{m}}, n_{\mathrm{r}}\right) & =\mathrm{e}^{-\beta\left[\frac{U}{2}\left(n_{\mathrm{m}}+n_{\mathrm{r}}\right)\left(n_{\mathrm{m}}+n_{\mathrm{r}}-1\right)-\mu_{i, \mathrm{~m}} n_{\mathrm{m}}-\mu_{i, \mathrm{r}} n_{\mathrm{r}}\right]},
\end{aligned}
$$

where $Z=\sum_{n_{\mathrm{m}}=0}^{2} \sum_{n_{\mathrm{r}}=0}^{4}\left(\begin{array}{c}2 \\ n_{\mathrm{m}}\end{array}\right)\left(\begin{array}{c}4 \\ n_{\mathrm{r}}\end{array}\right) B_{i}\left(n_{\mathrm{m}}, n_{\mathrm{r}}\right)$ is the partition function with local chemical potentials $\mu_{i, \mathrm{~m} / \mathrm{r}}=\mu_{\mathrm{m} / \mathrm{r}}-V_{i}$. Recalling that the correlator is the probability that the site $i$ and $j$ are singly occupied except for the case of the same spin component, we have

$$
\mathcal{P}_{i j}=\frac{1}{2} W_{i}(1,0) W_{j}(1,0)+\frac{3}{4} W_{i}(0,1) W_{j}(0,1)+W_{i}(1,0) W_{j}(0,1)+W_{i}(0,1) W_{j}(1,0),
$$

where the first term represents the case that both sites $i$ and $j$ are occupied by majority components $(\mathcal{N}=2$ in Eq. (3)), the second term for the case of minority components $(\mathcal{N}=4)$, and the other two terms for the cases that a site is occupied by a majority atom and the other site by a minority one. Figure S2 shows the calculated DPR for the imbalanced gas, together with the experimental results and the HTSE calculation. Although the increase in the calculated DPR due to the impurity components is clearly visible, it is still within the range of uncertainty of the experimental data. 


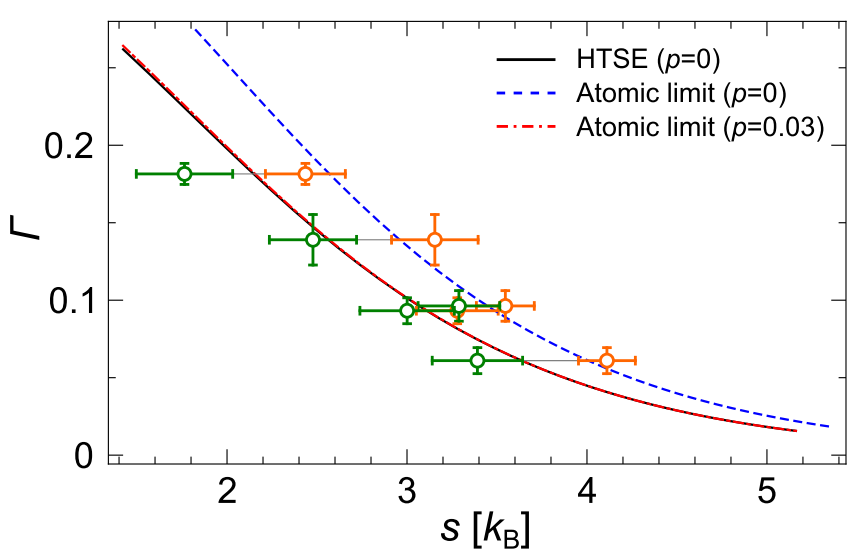

FIG. S 2. Effect of the impurity spin components on the doublon production rate. The experimental data (green and orange circles) and the HTSE calculation with $p=0$ (solid line) are identical with that shown as the $\mathrm{SU}(2)$ case in Figure $\mathrm{S} 1$ (b). The blue dashed line shows the atomic limit calculation with $p=0.03$ described in this section. Note that, the calculations on the HTSE and the atomic limit for $p=0$ (dash-dotted line) give almost the same result for the deep lattice $\left(V_{0}=11 E_{\mathrm{R}}\right)$ considered here. 
[1] Greif, D., Tarruell, L., Uehlinger, T., Jördens, R. \& Esslinger, T. Probing nearest neighbor correlations of ultracold fermions in an optical lattice. Phys. Rev. Lett. 106, 145302 (2011). 